\title{
Supported TritonX-100 Polyaniline Nano-Porous Electrically Active Film onto Indium-Tin-Oxide Probe for Sensors Application
}

\author{
Raju Khan \\ Analytical Chemistry Division, North East Institute of Science \& Technology, \\ Council of Scientific \& Industrial Research, Jorhat, India \\ E-mail:khan.raju@gmail.com \\ Received April 8, 2011; revised May 13, 2011; accepted May 29, 2011
}

\begin{abstract}
Supported tritonX100 polyaniline nano-porous electrically active film has been fabricated successfully onto indium-tin-oxide conducting probe using electrochemical polymerization process. The doping of TX-100 in the polymeric network of PANI was suggested using cyclic voltammeter, UV-vis spectroscopy, and Fourier Transform Infrared spectroscopy. The change in the surface morphology of PANI thin film due to incorporation of tritonX-100 was investigated using Atomic Forced Microscopy and porosity has been confirmed scanning electron microscopy, respectively. The surface morphology, uniformly disperse hexagonal close packing of TX-100 in PANI matrices due to the incorporation of TX-100 in polymeric network of PANI was confirmed by Atomic Force Microscopy. The electrical conductivity of PANI-TX-100 increases from $1.06 \times$ $10^{-2} \mathrm{~S} / \mathrm{cm}^{-1}$ to $4.95 \times 10^{-2} \mathrm{~S} / \mathrm{cm}^{-1}$ as the amount of TX-100 increases during the polymerization. The change in the morphology and electrical conductivity of PANI due to incorporation of TX-100 prove as a promising material for the sensing application.
\end{abstract}

Keywords: Electrochemical Polymerization, Non ionic surfactant, Conducting Polyaniline, Atomic Force Microscopy, Scanning Tunneling Microscopy

\section{Introduction}

The unique optical and electrical properties of conducting polymer make them a novel organic semi conducting material with great promise of their wide range of potential application includes storage batteries [1,2], electrochromic devices [3,4], light emitting diodes [5], non linear optics [6], and corrosion inhibitors [7] and sensors [8-11]. Among the conducting polymers, polyaniline (PANI), has attracted the interest of the researchers because of its good stability in air [12], simplicity of doping [13], improved electronic properties [14], electro chromic effect [15], well behaved electrochemical properties $[16,17]$ and moderately high conductivity in the doped state [18]. The changes in electrical and optical properties with interaction of oxidizing or reducing agents make them suitable for sensing applications. However, the difficult processability and poor thermal stability of PANI has to overcome for the successful application in sensors. In order to overcome this obstacle, different strategies for synthesis of PANI with colloids are a better alternate. Better processability may be achieved either by the synthesis on PANI in nano-micro scale particle, which are easier to disperse in a polymer matrix or by using an appropriate emulsifier which enhance the optical and electrical properties of PANI. Polymeric stabilizers (surfactant) affect the polymerization condition, kinetics, and also the final properties of the polymer $[19,20]$. Surfactants used as additives during the polymerization of aniline to effect the locus polymerization by using the emulsion [21,22] or inverse emulsion [23-25] pathways, and thus to modify the molecular and supermolecular structure of the resulting PANI, and to improve the properties of the PANI with respect to conductivity, stability, solubility in organic solvents, and processibility. Surfactants have important implications in wetting, formation of foams, etc., in addition to aggregation of surfactants at interfaces [26]. Polymeric nanostructures are formed on surfaces due to combination of interfacial, intra- and intermolecular forces [27]. 
Surfactants affect the preparation of PANI in three ways: 1) the presence of surfactant micellar and aqueous phases, thus altering the locus and the course of polymerization $[23,28], 2)$ anionic surfactants may act as counter ions for conducting-polymer polycations, and 3) the hydrophobic part of the surfactant molecules may adsorb on the produced conducting polymer, a surfactant thus becoming a part of the resulting material. The role of non-ionic surfactants, e.g. those based on poly (ethylene oxide) and Triton X-100 (TX-100) are less frequent and those dealing with cationic types are rare in the literature [29-31]. The nonionic surfactant Triton X-100 (TX-100) is a commercial product obtained by ethoxylation of p-(1,1,3,3-tetramethylbutyl) phenol and contains 9.5 oxyethylene units per molecule [32]. There are several theoretical [33-35] and experimental [31] studies concerning micellar properties of the TX100 system.

PANI with TritonX-100 chemical synthesis has been reported [36,37]. However, no attempts have been made electrochemically polymerization towards the preparation of conductive thin film of PANI with the nonionic surfactant i.e., Triton X-100. In this study, we are reporting electrochemical polymerized conductive thin film of PANI-Triton X-100, [4-(1,1,3,3-tetramethylbutyl) phenyl polyethylene glycol, TX-100]. The effect of the nonionic surfactant i.e., TX-100 on the electropolymerisation of aniline (An), at different surfactant ratios and potential windows is investigated. The doping of TX-100 changes the surface morphology of PANI. It is also found that electrical conductivity of PANI-TX-100 was better then that of PANI and governed by changing the concentration of surfactant during the polymerization of aniline. The present methods have been simpler, less expensive and more convenient route to electrochemically synthesize PANI nanostructures with tunable electrical properties.

\section{Experimental Section}

\subsection{Materials and Methods}

Hydrochloric acid (32\%) and aniline monomer (99.5\%) of analytical grade were purchased from Fluka, India, respectively. Nonionic surfactants i.e., TritonX-100 (TX$100,99 \%$ ) was purchased from Sigma Aldrich, USA. Other chemicals, $\mathrm{NaH}_{2} \mathrm{PO}_{4} \cdot \mathrm{H}_{2} \mathrm{O}, \mathrm{Na}_{2} \mathrm{HPO}_{4}, \mathrm{HCl}$, and $\mathrm{NaOH}$ were from Fisher. Aqueous solutions were prepared using analytical grade regents and $18 \mathrm{Mcm}$ - resistance water (NANO pure Diamond, Barnstead, Dubuque, IA). Sodium phosphate buffer solution (50 mM, pH 7.0, containing $0.9 \% \mathrm{NaCl}$ ) was prepared and used as common supporting electrolyte for cyclic voltametry experiments. Most measurements were carried out in a phos- phate buffer $(50 \mathrm{mM}, \mathrm{pH} 7.0$, containing $0.9 \% \mathrm{NaCl})$ supporting electrolyte medium. Electrochemical polymerization and cyclic voltametric measurements were carried out using Potentiostate/Glavan-ostat (Princeton Applied Research model).

\subsection{Thin Film Formation}

Polymerization of aniline on ITO electrode was carried out by electrochemical methods by applying an appropriate oxidation potential. In this study, chronoamperommetry method has been used for film formation by adjusting potential at -0.2 to $0.9 \mathrm{mV}$ in 150 seconds from solutions containing $0.2 \mathrm{M}$ aniline into $1 \mathrm{M} \mathrm{HCl}$ solution prepared in $10 \mathrm{~mL}$ of de-ionized water. Aniline in electrolyte was added to support electrochemical polymerization of TritonX-100 at different $(0.05,0.1,0.2$, and 0.4 $\mathrm{M}$ in $0.2 \mathrm{M}$ aniline based $1 \mathrm{M} \mathrm{HCl}$ solution). The active electrode surface area for sensor was $0.25 \mathrm{~cm}^{2}$ which was controlling by placing a physical mask during thin film formation.

\subsection{Surface Characterization}

The structural properties of electrochemically polymerized PANI-TX-100 films have been characterized using Fourier Transform Infrared spectrophotometer (Perkin Elmer) in the frequency range from $400-4000 \mathrm{~cm}^{-1}$. UV-visible spectrophotomer (Ocean Optics) in the wavelength range from $300-1000 \mathrm{~nm}$ is used to study the optical properties of deposited thin films. The surface morphology of thin film was investigated using Atomic Force Microscopy (AFM). Atomic Force Microscopy (AFM, Digital Instruments, Multi mode III) was used under the tapping mode. The surface conductivities of rectangular materials pallet were measured using four- point probe method by Kithley four-probe system with 224 programmable current source, 181 nano voltameter and 195A digital multimeter.

\section{Results and Discussion}

\subsection{Optical Properties}

The UV-visible absorption spectra of electrochemically polymerized thin film of PANI doped with $\mathrm{HCl}$ and TX-100 is shown in Figure 1(A). The absorption band at $320 \mathrm{~nm}$ is attributed to the л-л* transition within benzoid segment (excitation of the nitrogen atom in benzoid segment) and at $420 \mathrm{~nm}$ is related to the polaron band $\Omega^{*}$ transition in of PANI (protonation in PANI backbone, polaron/bipolaron transition that occurred in doped PANI). The absorption band at $850 \mathrm{~nm}$ is assigned to the 
л-localised polaron bands of doped PANI in its emaraldine salt form (conductive emaraldine salt state of PANI is represented by the letter two absorption bands) [8-11]. The absorption band at $320 \mathrm{~nm}$ and $440 \mathrm{~nm}$ are merged with the broadness in PANI-TX-100 and shifted towards the higher wavelength. The broadness and the intensity of merged absorption band increases on further increasing the amount of TX-100 during the polymerization. This may be due to the overlapping of absorption band due to the л-л* electronic transition in PANI and TX-100 and affinity of TX-100 with PANI matrices. This also may be due to the interaction of TX-100 with the benzoid ring system of PANI. This interaction of TX-100 with PANI will support conjugation (i.e., delocalization of electron in the polymeric network of PANI) and decrease the band gap of PANI. A little shift in the absorption band at $850 \mathrm{~nm}$ towards the lower wavelength is observed with the introduction of TX-100 into the polymeric network of PANI. The intensity of this absorption band is increases on further increasing the amount of TX-100. This shows that TX-100 stabilized the quinoid ring system of PANI and PANI-TX-100 is found in the doped state.

Figure 1(B) shows the infrared transmission spectrum of electrochemically polymerized thin film of PANI-HCl and PANI-TX-100 in the frequency range from 800 to $1700 \mathrm{~cm}^{-1}$. The infrared peak at 1570 and $1469 \mathrm{~cm}^{-1}$ are assigned to the non-symmetric vibration mode of $\mathrm{C}=\mathrm{C}$ in quinoid and benzenoid ring system in polyaniline respectively. The $\mathrm{C}-\mathrm{N}$ stretching vibration mode in aromatic amine nitrogen (quinoid system) in doped polyaniline is observed at $1297 \mathrm{~cm}^{-1}$, which is corresponds to the oxidation or protonation state of emareldine salt form $[9,10]$.
The absorption band at $1237 \mathrm{~cm}^{-1}$ is attributed to $\mathrm{C}-\mathrm{N}$ stretching vibration mode in benzenoid ring system of polyaniline assigned to conducted protonated form. The absorption band at $1156 \mathrm{~cm}^{-1}$ is assigned to in-plane vibration of $\mathrm{C}-\mathrm{H}$ bending vibration mode in $\mathrm{N}=\mathrm{Q}=\mathrm{N}$, $\mathrm{Q}-\mathrm{N}^{+} \mathrm{H}-\mathrm{B}$ or $\mathrm{B}-\mathrm{N}^{+} \mathrm{H}-\mathrm{B}$ (where $\mathrm{Q}=$ quinoid and $\mathrm{B}=$ benzenoid). This absorption band should occur during the polymerization i.e., polar structure of the conducted protonated form. The absorption bands at 884 and $816 \mathrm{~cm}^{-1}$ are attributed to the aromatic ring and out of plane $\mathrm{C}-\mathrm{H}$ deformation vibrations for 1,4-disubstituted aromatic ring system. The infrared spectrum of PANI-TX-100 consist the entire absorption band, markers of PANI along with the characteristics infrared band markers of TX-100 at 1600, 1460, 1351, 1298, 1246, 1184, 1124 and $953 \mathrm{~cm}^{-1}$. The infrared markers at $1246 \mathrm{~cm}^{-1}$ of PANI are retained in PANI-TX-100 with the sharp intensity. It means TX100 is doped inside the polymeric network of PANI and may enhance the properties of resulting polymer. The doping of TX-100 may also be proved by the breading of infrared absorption at $1124 \mathrm{~cm}^{-1}$ (corresponding to ether linkage in TX-100 PANI-TX-100. The small shift in the peak position and intensity might be due to the level of doping and nature of dopant in the resulting polymeric network of PANI. The shift in the peaks position of PANI towards the lower wavenumber by introducing the TX-100 is may due to the weak interaction forces (hydrogen bonding and vander Waal forces etc.) between TX-100 and PANI matrices. TX-100 supports the polymeric network of PANI this is proving by the shift in the absorption bands at 884 and $816 \mathrm{~cm}^{-1}$ towards the higher wave number.
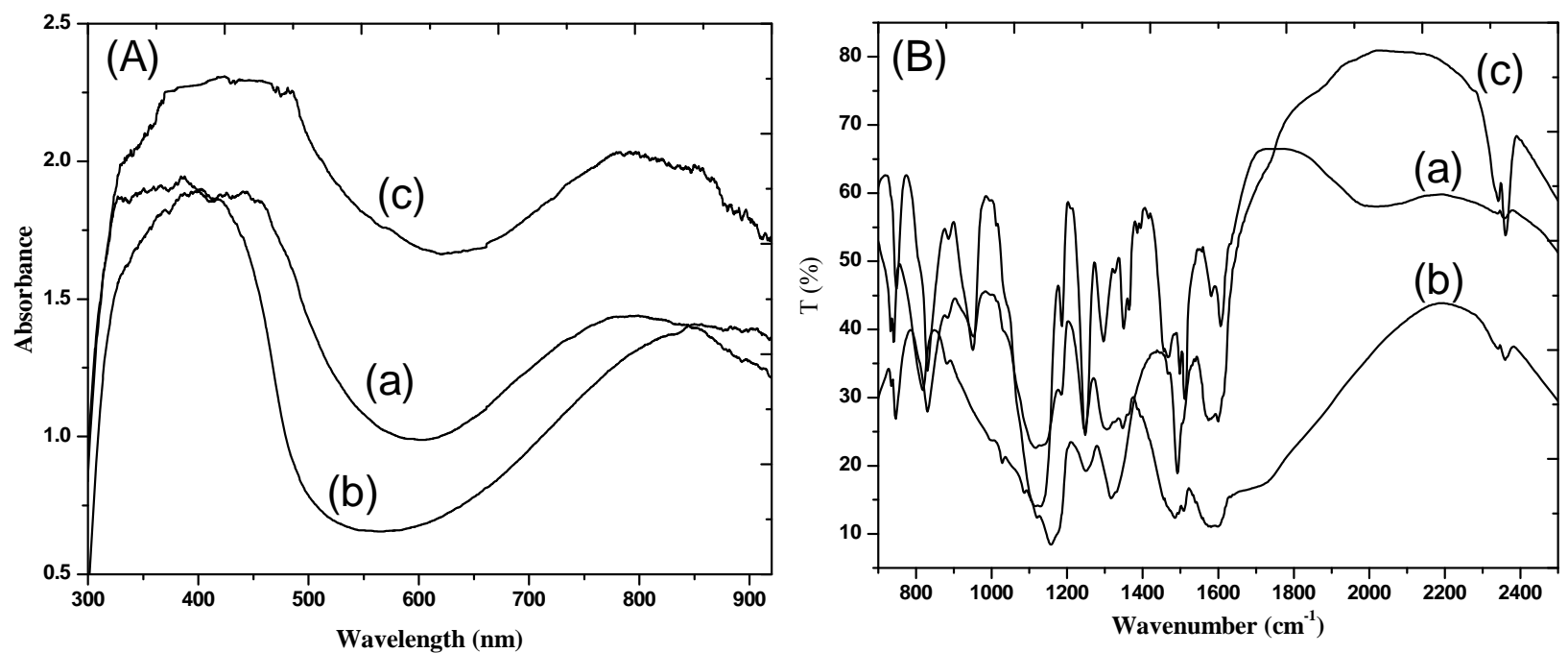

Figure 1. (A) UV-vis spectra electrochemically polymerized thin film of (a) PANI-HCI/ITO (b) PANI-TX100/ITO (0.05M) and (c) PANI-TX100/ITO (0.1M) and (B) FTIR spectra electrochemically polymerized thin film of (a) PANI-HCI/ITO (b) PANI-TX100/ITO (0.05M) and (c) PANI-TX100/ITO (0.1M). 


\subsection{Atomic Force Microscopy}

The surface morphology of electrochemically deposited thin film is shown in Figure 2(a) PANI (b) PANITX-100 (0.05M) and (c) PANI-TX-100 (0.1M) investigated using AFM. The AFM micro images shows that TX-100 are uniformly dispersed as hexagonal closed packing into the PANI matrices and TX-100 phase and PANI phase are found to be strictly interconnected with no major macroscopic phase separation. This proves that incorporation of TX-100 change the surface morphology of PANI i.e., plane morphology changes into the rough surface. This changed morphology of PANI with embadation of TX-100 in PANI matrices may use in sensing applications.

\subsection{Scanning Electron Microscopy}

The surface morphologies of (a) PANI/ITO, (b) PANI-TX100/ITO (0.05M) electrode and (c) PANITX100/ITO (0.1M) electrodes have been investigated using Scanning Electron Microscopy (SEM) respectively. SEM of PANI film shows porous non-uniform sponge like, rough structure (Figure 3(a)) wherein, a new regular cage like network appears after the introduction of TX-100 in PANI backbone (Figures 3(b) and (c)) suggesting that TX-100 is uniformly distributed in PANI matrix. After the increase of TX-100, cage like morphology of PANI-TX-100 has been changes into another regular uniform porosity form resulting due to coverage of available active cites on PANI film surface by TX-100. It may be noted that the affinity of TX-100 is very strong with PANI and its incorporation in PANI. The porous surface morphology has suitable advantage for enzymes and antibodies immobilizations are expected to adsorb strongly on the surface of PANI-TX-100.

\subsection{Electrical Conductivity Measurements}

The results of electrical conductivity electrochemically polymerized PANI thin film prepared in the presence of nonionic surfactants TX-100 are summarized in Table 1. An increase in the electrical conductivity of PANI is observed on further increasing the concentration of TX-100 during the polymerization of aniline. It's suggested the electrochemical process for PANI is based on radical cation intermediate coupling and the surfactant molecules provide a hydrophobic effect preventing the polymer degradation, and thus improving the degree of structural order of PANI film [38]. The TX-100 may neutralize or stabilized the polymer chain that may enhance the polarons transfer, which leads to increase in conductivity. Thus the increase in the electrical conductivity of PANI -TX-100 is due to the doping of TX-100 in the polymeric network of PANI that may support the delocalization of electron within the PANI metrices. This change in the electrical conductivity with concentration of dopant proves that TX-100 doped PANI may be used as a sensing material.
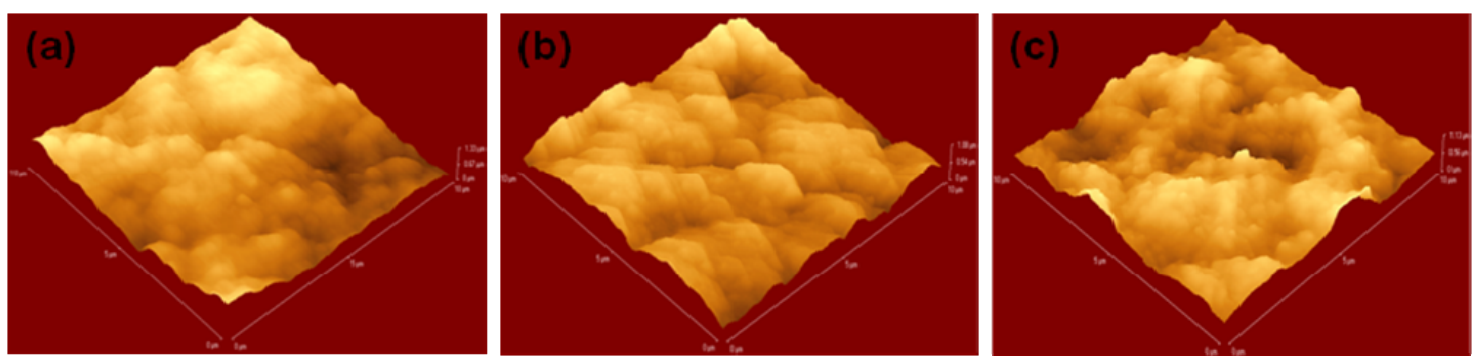

Figure 2. Atomic force microscopic images of (a) PANI-HCI/ITO (b) PANI-TX100/ITO (0.05M) and (c) PANI-TX100/ITO (0.1M)
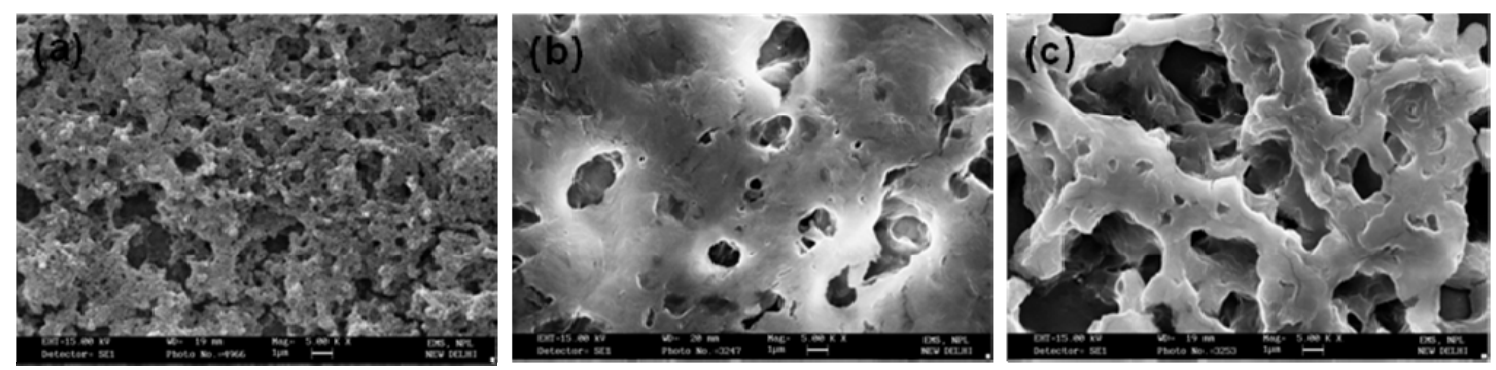

Figure 3. Scanning electron microscopy images of (a) PANI-HCI/ITO (b) PANI-TX100/ITO (0.05M) and (c) PANITX100/ITO (0.1M). 
Table 1. Electrical conductivity of PANI and PANI-TX-100 thin film on to indium-tin-oxide probe.

\begin{tabular}{ccccc}
\hline S. No. & Matrix & Added (TX-100) & $\begin{array}{c}\text { Temperature } \\
\left({ }^{\circ} \mathrm{C}\right)\end{array}$ & Electrical conductivity \\
\hline 1 & PANI-HCl & No TX-100 & $30^{\circ} \mathrm{C}$ & $2.36 \times 10^{-3} \mathrm{~S} / \mathrm{cm}$ \\
2 & PANI-TX-100 & $0.05 \mathrm{M}$ & $30^{\circ} \mathrm{C}$ & $1.06 \times 10^{-2} \mathrm{~S} / \mathrm{cm}$ \\
3 & PANI-TX-100 & $0.1 \mathrm{M}$ & $30^{\circ} \mathrm{C}$ & $1.38 \times 10^{-2} \mathrm{~S} / \mathrm{cm}$ \\
4 & PANI-TX-100 & $0.2 \mathrm{M}$ & $30^{\circ} \mathrm{C}$ & $2.59 \times 10^{-2} \mathrm{~S} / \mathrm{cm}$ \\
5 & PANI-TX-100 & $0.4 \mathrm{M}$ & $30^{\circ} \mathrm{C}$ & $4.95 \times 10^{-2} \mathrm{~S} / \mathrm{cm}$ \\
\hline
\end{tabular}

\subsection{Cyclic Voltammogram Analysis}

In the Figure 4 shows CV of PANI and PANI-TX-100 matrix at PBS $(50 \mathrm{mM}, \mathrm{pH} 7.0$, containing $0.9 \% \mathrm{NaCl})$ at constant $30 \mathrm{mVs}^{-1}$ scan rate. The PANI layer was seen to be redox active in the potential region -200 to $+900 \mathrm{mV}$ was studied, exhibiting three sets of redox peaks. Initially, the oxidation of aniline occurred at approximately $+800 \mathrm{mV}$ resulting due to nucleation of PANI. During subsequent scans the oxidation of aniline occurred at lower potentials due to the catalytic effect of PANI, which resulted in deposition of polymer on the electrode surface. Redox couples peaks were attributed to intrinsic redox processes of the polymer itself. The redox couple peak occurred at approximately $+200 \mathrm{mV}$ and is attributed to the transformation of PANI from the reduced leucoemeraldine (LE) state to the partially oxidized emeraldine state (EM). The redox couple peak at approximately $+800 \mathrm{mV}$ corresponds to transition of the PANI from LE to pernigraniline (PE) state, and is accompanied by the oxidation of aniline monomer [39]. The redox couple peak at approximately $+500 \mathrm{mV}$, which is geneally attributed to the redox reaction of p-benzoquinone

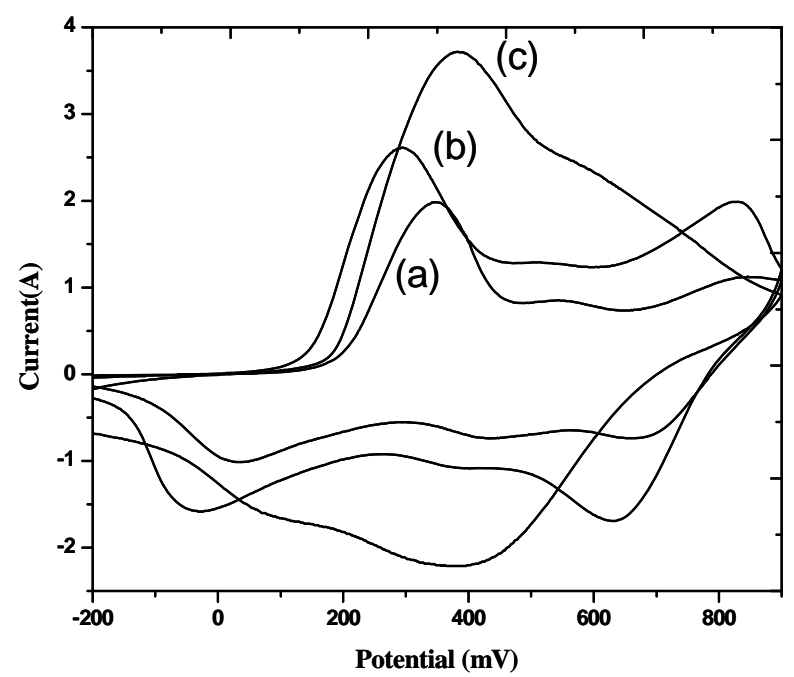

Figure 4. Cyclic Voltammogram of (a) PANI-HCI/ITO (b) PANI-TX100/ITO (0.05M) and (c) PANI-TX100/ITO (0.1M) using as supporting electrolyte PBS (50mM, pH 7.0, containing $0.9 \% \mathrm{NaCl}$ ).
[40], is less intense. The cathodic and anodic peak positions of PANI-TX-100 thin film shifted and increase the charge transfer appreciably corresponding to PANI-HCl are shown in the curve (b). This increase in current was due to fast redox process at PANI-TX-100 matrix surface. Difference between cathodic (Epc) and anodic peak (Epa) shift $(\Delta \mathrm{Ep}=\mathrm{Epa}-\mathrm{Epc})$ was use to determine the kinetics of electron transfer in these matrixes. This may be due to electrostatic interactions between the head group of TX-100 and charged aniline at the hydrophilic interface of the micelles.

\section{Conclusions}

Electrochemical polymerization process was used for stable thin film deposition of TX-100 with PANI. TX100 molecules in PANI had enhanced charge transfer and current voltage characteristics at electrode interface. Increases in oxidation peak current and enhanced broadening were observed due to fast redox process in PANITX-100 matrix. UV-vis and FTIR spectroscopy confirms the interaction of TX-100 in PANI matrices. The electrical conductivity of PANI-TX-100 thin film increases on further increasing the concentration of TX-100 during the polymerization of aniline. AFM and SEM studies show the incorporation of TX-100 in PANI matrices that changes the surface morphology of PANI. The change in surface morphology and electrical conductivity of PANITX-100 with the concentration of TX-100 shows the potential for sensing application. The electrical conductivity of PANI-TX-100 increases from $1.06 \times 10^{-2} \mathrm{~S} / \mathrm{cm}^{-1}$ to $4.95 \times 10^{-2} \mathrm{~S} / \mathrm{cm}^{-1}$ as the amount of TX-100 increases during the polymerization. The electrochemically synthesized PANI-TX-100 thin film enhances the electrical conductivity of PANI film and make suitable for sensing application.

\section{Acknowledgements}

Raju Khan is thankful to the Department of Science and Technology (DST), Government of India for financial support received under the awarded Fast Track Young scientist project No. SR/FTP/CS-77/2007. 


\section{References}

[1] H. Yamamaoto, M. Oshima, T. Hosaka and I. Isa, "Solid Electrolytic Capacitors Using an Aluminum Alloy Electrode and Conducting Polymers," Synthetic Metals, Vol. 104, No. 1, 1999, pp. 33-38. doi:10.1016/S0379-6779(99)00003-X

[2] K. S. Ryu, K. M. Kim, S. G. Kang, G. J. Lee, J. Joo and S. H. Chang, "Electrochemical and Physical Characterization of Lithium Ionic Salt Doped Polyaniline as a Polymer Electrode of Lithium Secondary Battery," Synthetic Metals, Vol. 110, No. 3, 2000, pp. 213-217. doi:10.1016/S0379-6779(99)00288-X

[3] A. Talei, J. Y. Lee, Y. K. Lee, J. Jang, J. A. Romagnoli, T. Taguchi and E. Maeder, "Dynamic Sensing Using Intelligent Composite: An Investigation to Development of New Ph Sensors and Electrochromic Devices," Thin Solid Films, Vol. 363, No. 1-2, 2000, pp. 163-166. doi:10.1016/S0040-6090(99)00987-6

[4] A. Kumar, D. M. Welsh, M. C. Morvant, F. Piroux, K. A. Abboud and J. R. Reynolds, "Conducting Poly (3,4- alkylenedioxythiophene) Derivatives as Fast Electrochromics with High-Contrast Ratios," Chemistry of Materials, Vol. 10, No. 3, 1998, pp. 896-902. doi: $10.1021 / \mathrm{cm} 9706614$

[5] J. C. Scott, S. A. Carter, S. Karg and M. Angelopoulos, "Polymeric Anodes for Organic Light-Emitting Diodes," Synthetic Metals, Vol. 85, No. 1-3, 1997, pp. 1197-1200. doi:10.1016/S0379-6779(97)80207-X

[6] Y. T. Shin, S. W. Shin, J. Shin, K. Lee and M. Cha, "Pulsed Laser Deposition of a Thin Conjugated-Polymer Film," Thin Solid Films, Vol. 360, No. 1-2, 2000, pp. 13-16. doi:10.1016/S0040-6090(99)00962-1

[7] B. Wessling and J. Posdorfer, "Corrosion Prevention with an Organic Metal (polyaniline): Corrosion Test Results," Electrochimica Acta, Vol. 44, No. 12, 1999, pp. 21392147. doi:10.1016/S0013-4686(98)00322-3

[8] R. Khan and M. Dhayal, "Chitosan/Polyaniline Hybrid Conducting Biopolymer Base Impedimetric Immunosensor to Detect Ochratoxin-A," Biosensors \& Bioelectronics, Vol. 24, No. 6, 2009, pp. 1700-1705. doi:10.1016/j.bios.2008.08.046

[9] A. A. Ansari, R. Khan, K. N. Sood and B. D. Malhotra, "Polyaniline-Cerium Oxide Nanocomposite for Hydrogen Peroxide Sensor," Journal of Nanoscience \& Nanotechnology, Vol. 9, No. 8, 2009, pp. 4679-4685. doi:10.1166/jnn.2009.1085

[10] A. Kaushik, R. Khan, V. Gupta, B. D. Malhotra and S. P. Singh, "Hybrid Cross Linked Polyaniline-WO3 Nanocomposite Thin Films for $\mathrm{NO}_{\mathrm{x}}$ Gas Sensing," Journal of Nanoscience \& Nanotechnology, Vol. 9, 2009, pp. 17921796. doi:10.1166/jnn.2009.417

[11] A. Kaushik, J. Kumar, M. K. Tiwari, R. Khan, B. D. Malhotra, V. Gupta and S. P. Singh, "Fabrication and Characterization of Polyaniline-ZnO Hybrid Nanocomposite Thin Films," Journal of Nanoscience \& Nanotechnology, Vol. 8, No. 4, 2008, pp. 1757-1761. doi:10.1166/jnn.2008.006
[12] B. Wesseling, "Electrical Properties of Pyrrole and Its Copolymers," Synthetic Metals, Vol. 4, No. 2, 1991, pp. 119-130.

[13] Y. Z. Zheng, K. Levon, T. Taka, J. Laasko and J. E. Osterholm, "Doping-induced Layered Structure in Nalkylated Polyanilines," Polymer Journal, Vol. 28, No. 5, 1996, pp. 412-418. doi:10.1295/polymj.28.412

[14] Y. Cao, P. Smith, A. J. Heeger, PCT, Patent Application WO 92/2291, Vol. 91, 1992.

[15] Y. Xia, A. G. MacDiarmid and A. J. Epstein, "Camphorsulfonic Acid Fully Doped Polyaniline Emeraldine Salt: In situ Observation of Electronic and Conformational Changes Induced by Organic Vapors by an Ultra- violet/ isible/Near-Infrared Spectroscopic Method," Maomocules, Vol. 27, No. 24, 1994, pp. 7212-7214. doi: $10.1021 / \mathrm{ma} 00102 \mathrm{a} 033$

[16] A. G. MacDiarmid and A. J. Epstein, "The Concept of Secondary Doping as Applied to Polyaniline," Synthetic Metals, Vol. 65, 1994, pp. 103-116. doi:10.1016/0379-6779(94)90171-6

[17] A. G. MacDiarmid, Y. N. Xia and J. M. Wiesinger, U.S. Patent 5, 773, 568 (1998).

[18] A. G. MacDiarmid and A. J. Epstein, "Secondary Doping in Polyaniline" Synthetic Metals, Vol. 69, No. 1-3, 1995, pp. 85-92. doi:10.1016/0379-6779(94)02374-8

[19] S. P. Armes and M. Aldissi, "Novel Colloidal Dispersons of Polyaniline," Journal of the Chemical Society, Chemical Communication, Vol. 2, 1989, pp. 88-89. doi:10.1039/c39890000088

[20] N. Kohut-Svelko, S. Reynaud and J. Francois, "Synthesis and Characterization of Polyaniline Prepared in the Presence of Nonionic Surfactants in an Aqueous Disrsion," Synthetic Metals, Vol. 150, No. 2, 2005, pp. 107-14. doi:10.1016/j.synthmet.2004.12.022

[21] P. J. Kinlen, J. Liu, Y. Ding, C. R. Graham and E. E. Remsen, "Emulsion Polymerization Process for Organically Soluble and Electrically Conducting Polyaniline," Macromolecules, Vol. 31, No. 6, 1998, pp. 1735-1744. doi:10.1021/ma9714301

[22] S. Palaniappan, "Preparation of Polyaniline-Sulfate Salt by Emulsion and Aqueous-Polymerization Pathway Without Using-Protonic Acid," Polymers for Advanced Technologies, Vol. 13, No. 1, 2002, pp. 54-59. doi:10.1002/pat.154

[23] H. Xia and Q. Wang, "Synthesis and Characterization of Conductive Polyaniline Nanoparticles Through U1trasonic Assisted Inverse Microemulsion Poly-mezation," Journal of Nanoparticle Research, Vol. 3, 2001, pp. 40141.

[24] P. S. Rao, S. Subrahmanya and D. N. Sathyanarayana, "Inverse Emulsion Polymerization: A New Route For the Synthesis of Conducting Polyaniline," Synthetic Metals, Vol. 128, No. 3, 2002, pp. 311-316. doi:10.1016/S0379-6779(02)00016-4

[25] P. S. Rao, D. N. Sathyanarayana and S. Palaniappan, "Polymerization of Aniline in an Organic Peroxide System by the Inverted Emulsion Process," Macromolecules, Vol. 35, No. 13, 2002, pp. 4988-4996. 
doi:10.1021/ma0114638

[26] D. W. C. Andrew, E. A. O' Rear and B. P. Grady, “Adsorbed Surfactants as Templates for the Synthesis of Morphologically Controlled Polyaniline and Polypyrrole Nanostructures on Flat Surfaces: From Spheres to Wires to Flat Films," Journal of the American Chemical Society, Vol. 125, No. 48, 2003, pp. 14793-14800. doi: $10.1021 / \mathrm{ja} 0365983$

[27] J. P. Rabe, "Self-assembly of Single Macromolecules at Surfaces," Current Opinion in Colloid \& Interface Science, Vol. 3, No. 1, 1998, pp. 27-31. doi:10.1016/S1359-0294(98)80038-1

[28] D. Ichinohe, T. Aria and H. Kise, "Synthesis of Soluble Polyaniline in Reversed Micellar Systems," Synthetic Metals, Vol. 84, No. 1-3, 1997, pp. 75-76. doi:10.1016/S0379-6779(96)03843-X

[29] D. Kim, J. Choi, J.-Y. Kim, Y.-K. Han and D. Sohn, "Size Control of Polyaniline Nanoparticle by Polymer Surctant, Macromolecules, Vol. 35, No. 13, 2002, pp. 5314-316. doi:10.1021/ma020162a

[30] M. Omastova, M. Trchova, J. Kovarova and J. Stejskal, "Synthesis and Structural Study of Polypyrroles Prepared in the Presence of Surfactants," Synthetic Metals, Vol. 138, No. 3, 2003, pp. 447-455. doi:10.1016/S0379-6779(02)00498-8

[31] Z. Zhang, Z. Wei and M. Wan, "Nanostructures of Polyiline Doped with Inorganic Acids," Mcromolecules, Vol. 35, No. 15, 2002, pp. 5937-5942.

[32] A. N. Galatanu, I. S. Chrorrakis, D. F. Anghel and A. Khan, "Ternary Phase Diagram of the Triton X-100/Poly acrylic acid)/Water System," Langmuir, Vol. 16, No. 11, 2000, pp. 4922-4928. doi:10.1021/la991668y

[33] R. J. Robson and E. A. Dennis, "The Size, Shape, and
Hydration of Nonionic Surfactant Micelles Triton X100," The Journal of Physical Chemistry, Vol. 81, No. 11, 1977, pp. 1075-1077. doi:10.1021/j100526a010

[34] C. Tanford, Y. Nozaki and M. F. Rhode, "Size and Shape of Globular Micelles Formed in Aqueous Solution by NAlkyl Polyoxyethylene Ethers," The Journal of Physical Chemistry, Vol. 81, No. 18, 1977, pp. 1555-1560. doi:10.1021/j100531a007

[35] A. A. Ribeiro and E. A. Dennis, "Proton Magnetic Resonance Relaxation Studies on The Structure of Mixed Micelles of Triton X-100 and Dimyristoylphosphatidylcholine," Biochemistry, Vol. 14, No. 17, 1975, pp. 37463755. doi:10.1021/bi00688a005

[36] T. C. Girija and M. V. Sangarranarayanan, "PolyanilineBased Nickel Electrodes For Electrochemical Supercapacitors-Influence of Triton X-100," Journal of Power Sources, Vol. 159, No. 2, 2006, pp. 1519-1526. doi:10.1016/j.jpowsour.2005.11.078

[37] N. Arsalani, M. Khavei and A. A. Entezami, "Synthesis and Characterization of Novel NSubstituted Polyaniline by Triton X-100," Iranian Polymer Journal, Vol. 12, No. 3, 2003, pp. 237-242.

[38] L.T. Cai, S. B. Yao and S. M. Zhou, "Surfactant Effects on the Polyaniline Film," Synthetic Metals, Vol. 88, No. 3, 1997, pp. 209-212. doi:10.1016/S0379-6779(97)03852-6

[39] K.R. Prasad and N. Munichandraiah, "Potentiodynamic Deposition of Polyaniline on Non-Platinum Metals and Characterization," Synthetic Metals, Vol. 123, No. 3, 2001, pp. 459-468. doi:10.1016/S0379-6779(01)00334-4

[40] J. C. Cooper and E. A. H. Hall, "Electrochemical Response of an Enzyme-Loaded Polyaniline Film," Biosens \& Bioelectronics, Vol. 7, No. 7, 1992, pp. 473-485. 